International Journal of Pure and Applied Mathematics

Volume 107 No. 4 2016, 821-843

ISSN: $1311-8080$ (printed version); ISSN: 1314-3395 (on-line version)

url: http://www.ijpam.eu

doi: 10.12732 /ijpam.v107i4.3

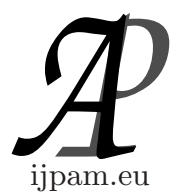

\title{
A SYMBOLIC APPROACH TO NONLINEARLY PERTURBED HEAT EQUATION
}

\author{
Carlos Correia Ramos ${ }^{1}$, Ana Isabel Santos ${ }^{2}$, Sandra Vinagre ${ }^{3} \S$ \\ ${ }^{1,2,3}$ Department of Mathematics, CIMA \\ University of Évora \\ Rua Romão Ramalho 59, 7000-671, Évora, PORTUGAL
}

\begin{abstract}
We consider a system described by the linear heat equation with adiabatic boundary conditions which is perturbed periodicaly. This perturbation is nonlinear and is characterized by a one-parameter family of quadratic maps. The system, depending on the parameters, presents very complex behaviour. We introduce a symbolic framework to analyze the system and resume its most important features.
\end{abstract}

AMS Subject Classification: 37B10, 37B40, 39B12, 35K05, 37K60, 37E05, 74H65

Key Words: heat equation, chaotic dynamics, symbolic dynamics, iteration theory, topological entropy, complex systems

\section{Introduction and Preliminaries}

We consider an ideal system consisting on the temperature changes on a wire with adiabatic endpoints. When the system is isolated evolves continuously, determined by the linear heat equation, and in this regime we have the explicit time dependent solution. We consider that the system is not permanently isolated, instead is periodically perturbed. When the perturbation disappears the system evolves again continuously and the process repeats itself after constant time intervals. The perturbation is modeled by a nonlinear iterated interval map in a manner described below in detail.

Received: November 23, 2015

Published: May 4, 2016

${ }^{\S}$ Correspondence author
(C) 2016 Academic Publications, Ltd.

url: www.acadpubl.eu 
The physical context is introduced as a motivation for the study of certain infinite dimensional systems which have, nonetheless, much properties strongly related with iteration of interval maps (one dimensional systems). These infinite dimensional dynamical systems, despite its simple definition, may present very complex behaviour showing some features of real turbulence regarding the balance between coherence versus disorder. In particular, depending on the parameters, the systems present chaotic behaviour, that is, non-periodic evolution and sensitivity to initial conditions, with exponential divergence of two arbitrary close initial temperature functions. These type of systems were first introduced in [1]. On the other hand, iteration in function spaces were previously studied in the papers of Severino et al. [2], [3] and [4], Sharkovsky [5] and Vinagre et al. [6]. It is considered a certain space $\mathcal{A}$ and an operator $T_{f}$, induced by an interval map $f$, so that for $\varphi \in \mathcal{A}$ we have $T_{f}(\varphi)=f \circ \varphi$. The particular feature of the system $\left(\mathcal{A}, T_{f}\right)$, for certain choices of the map $f$, is that the number of different critical values will always grow with the iteration of $T_{f}$, for almost all initial conditions. This is an essential aspect of the notion of ideal turbulence introduced by Sharkovsky [7] and [8]. In fact, systems such $\left(\mathcal{A}, T_{f}\right)$, for certain choices of $f$, presents ideal turbulence and its main characteristic is a cascade process which produce structures of arbitrarily small scale, meaning that in the limit the functions, under iteration, become fractal functions or even stochastic functions, depending on the ergodic properties of the map $f$.

On the contrary, for the system presented here and in our previous work [1], the number of critical points does not grow exponentially. During an initial transient time interval the number of critical points do grow exponentially, however, quickly the system attains a balance between the creation of new critical points due to the interval map effect, and the destruction of critical points due to the dissipative effect of the heat equation.

In the present work we introduce a discretization of the state space and search for evolution rules similar to cellular automata, or eventually substitutional systems. We did not find any simple deterministic rule, nevertheless the symbolic framework introduced here turned out to be very usefull to characterize different aspects of the global dynamics. Namelly, the distribution and evolution of the critical points and their positions with respect to the intervals of monotonicity, which constitutes the basin of atraction of the interval map $f_{\mu}$ defined below. The introduced codification is precisely based on the atractor of the quadratic map.

If $\mu$ is so that the map has positive topological entropy then, although there are no deterministic rules for the symbolic flow, there is a statistical structure which depends on the parameters $\mu$ and $\lambda$. This statistical structure 
is revealed in the fixed frequence of ocurrence of symbols, not depending on the initial conditions. We determined an empirical relation which characterizes this phenomena. If $\mu$ is less than the Feigenbaum constant, therefore with zero topological entropy, several unexpect phenomena occurs. Namely, the existence of atracting periodic orbits for almost every initial conditions, that is, for almost every initial condition the limit is a periodic orbit. Moreover, the referred structure of frequence of symbol occuring is no longer valid and there is no empirical relation in this case.

Before giving the definition in detail of the system, we present some preliminaries and discussion related with previous work.

Consider a $m$-modal map $f$ in the class $C^{1}(I, I)$, for a certain interval $I$, and the class of differentiable functions

$$
\mathcal{A}=\left\{\varphi \in C^{1}([0,1]): \varphi^{\prime}(0)=\varphi^{\prime}(1)=0,|c p(\varphi)|<\infty\right\},
$$

where $|c p(\varphi)|$ denotes the number of critical points of $\varphi$. That is, a function belongs to the class $\mathcal{A}$ if it is differentiable, its derivatives at the endpoints are 0 and its number of critical points are finite. Consider also the operator $T_{f}$ defined by

$$
\begin{aligned}
T_{f}: \mathcal{A} & \rightarrow \mathcal{A} \\
\varphi & \mapsto f \circ \varphi .
\end{aligned}
$$

Note that this operator is well defined since $(f \circ \varphi)^{\prime}(0)=(f \circ \varphi)^{\prime}(1)=0$. Moreover, if $\phi \in \mathcal{A}$ and $\operatorname{Im}(\phi) \subset I$, then $\operatorname{Im}\left(T_{f}^{k} \phi\right) \subset I$, for every $k \in \mathbb{N}$. Therefore, we obtain a discrete infinite dimension dynamical system $\left(\mathcal{A}, T_{f}\right)$ in the sense that we have a set $\mathcal{A}$ (with additional structure, a topology or a metric, for now not specified) and a self map $T_{f}$, which characterizes the discrete time evolution.

The considered interval maps, modeling the perturbation, belongs to the well studied quadratic family defined by $f_{\mu}(x)=1-\mu x^{2}$, with $\mu \in(0,2]$. There is a maximal invariant interval, $[-1,1]$, where the relevant dynamics occurs, that is, the iterates $f_{\mu}^{k}\left(x_{0}\right):=f_{\mu}\left(\ldots f_{\mu}\left(x_{0}\right)\right)$ ( $k$ times) of initial points $x_{0}$ in $[-1,1]$ will belong to $[-1,1]$, for every $k$. For initial points $x_{0}$ outside $[-1,1]$, the iterates $f_{\mu}^{k}\left(x_{0}\right)$, for $k \in \mathbb{N}$, diverge to infinity. Therefore, we consider the one parameter family of operators $T_{f_{\mu}}$, induced by $f_{\mu}$ on $\mathcal{A}$. Next, we show an example with several iterates under $T_{f_{\mu}}$ of a given initial function $\phi_{0}$.

Example 1. Consider $f_{\mu}(x)=1-\mu x^{2}$, with $\mu=2$ and

$$
\begin{aligned}
\phi_{0}(x)= & 0.2+0.1 \cos (\pi x)-0.2 \cos (2 \pi x)+0.1 \cos (3 \pi x)+ \\
& +0.1 \cos (4 \pi x)-0.1 \cos (5 \pi x)+0.2 \cos (6 \pi x) .
\end{aligned}
$$




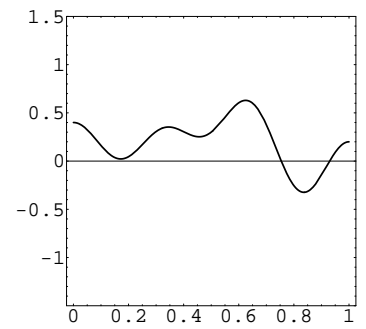

(a)

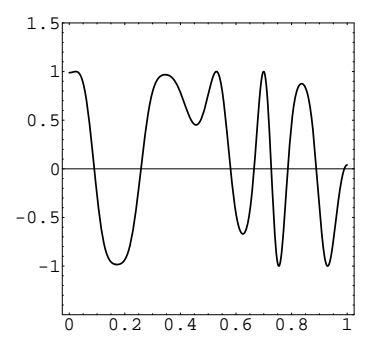

(d)

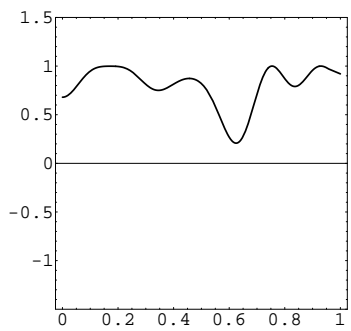

(b)

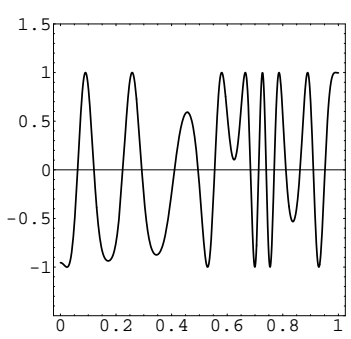

(e)

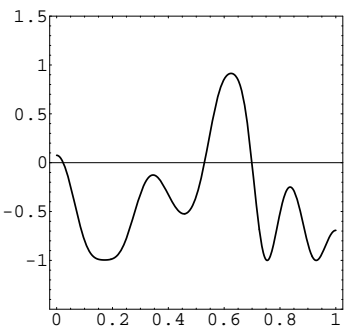

(c)

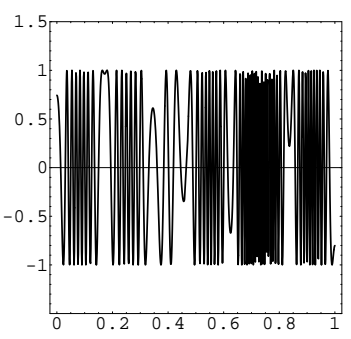

(f)

Figure 1: Graphs of (a) $\phi_{0}(x),(\mathrm{b}) \phi_{1}(x),(\mathrm{c}) \phi_{2}(x),(\mathrm{d}) \phi_{3}(x)$, (e) $\phi_{4}(x)$ and (f) $\phi_{7}(x)$, with $f_{\mu}(x)=1-\mu x^{2}, \mu=2$ and $\phi_{0}(x)=0.2+$ $0.1 \cos (\pi x)-0.2 \cos (2 \pi x)+0.1 \cos (3 \pi x)+0.1 \cos (4 \pi x)-0.1 \cos (5 \pi x)+$ $0.2 \cos (6 \pi x)$.

In the Figure 1, we can see increasing frequencies and the asymptotic behaviour expresses the occurrence of ideal turbulence.

\section{Symbolic Dynamics for Unimodal Maps}

Consider a unimodal map $f$ in the interval $I=[-1,1]$, with 0 being the unique critical point where $f$ gets the maximal value. Assume that $f \in C^{1}(I, I)$. We assign the symbols $L$ (left) and $R$ (right) to each point $x$ of the subintervals of monotonicity $[-1,0)$ and $(0,1]$, respectively, and the symbol $C$ to the critical point 0 . This assignment is called the address of $x$ and it is denoted by $a d(x)$. 
The address of the point $x, a d(x)$, is thus given by

$$
\operatorname{ad}(x)=\left\{\begin{array}{lll}
L & \text { if } & x<0 \\
C & \text { if } & x=0 \\
R & \text { if } & x>0
\end{array}\right.
$$

As usual, we get a correspondence between orbits of points and symbolic sequences of the alphabet $\{L, C, R\}$, the itinerary of $x$ under the map $f$,

$$
i t_{f}(x):=\operatorname{ad}(x) \operatorname{ad}(f(x)) \operatorname{ad}\left(f^{2}(x)\right) \cdots \in\{L, C, R\}^{\mathbb{N}} .
$$

Let $I_{S_{0} S_{1} \ldots S_{k}} \subset[-1,1]$ be the interval of points $x$ defined by

$$
\begin{aligned}
I_{S_{0} S_{1} \ldots S_{k}} & =\left\{x \in I: x \in I_{S_{0}}, f(x) \in I_{S_{1}}, \ldots, f^{k}(x) \in I_{S_{k}}\right\} \\
& =I_{S_{0}} \cap f^{-1}\left(I_{S_{1}}\right) \ldots f^{-k}\left(I_{S_{k}}\right)
\end{aligned}
$$

where $S_{j}=a d\left(f^{j}(x)\right), j=0,1, \ldots, k$, and $f^{-j}\left(I_{S_{j}}\right)$ denotes the pre-image of the interval $I_{S_{j}}, j=1, \ldots, k$.

The kneading sequence of an unimodal map $f$ is the itinerary of the image of the critical point, that is

$$
\mathcal{K}:=i t_{f}(f(0))=K_{1} K_{2} \cdots \in\{L, C, R\}^{\mathbb{N}}
$$

(see [9]).

An admissible sequence is a sequence in $\{L, C, R\}^{\mathbb{N}}$ which occurs as an itinerary for some point $x \in[-1,1]$ and an admissible word is some word occurring in an admissible sequence. The sequence space is the set of all infinite admissible sequence in $\{L, C, R\}^{\mathbb{N}}$ and is denoted by $\Sigma$.

In the sequence space $\Sigma$, we define the usual shift map $\sigma: \Sigma \rightarrow \Sigma$ by

$$
\sigma\left(P_{1} P_{2} P_{3} \ldots\right)=P_{2} P_{3} \ldots
$$

where $P_{1} P_{2} P_{3} \cdots \in \Sigma$, and we have

$$
\sigma\left(i t_{f}(x)\right)=i t_{f}(f(x))
$$

Therefore, we obtain the symbolic system $(\Sigma, \sigma)$ associated with the unimodal map. 


\section{Nonlinear Perturbed Heat Equation}

We consider the unit interval representing an ideal wire. The temperature function at each point $x \in[0,1]$ and each time instant $t \in \mathbb{R}_{0}^{+}$is denoted by $\psi(x, t)$. We consider also that the wire is such that the time evolution of the temperature function is described by the linear heat equation

$$
\frac{\partial \psi}{\partial t}=\lambda \frac{\partial^{2} \psi}{\partial x^{2}},
$$

where $\lambda$ is a constant, the diffusion coefficient. If there is no heat exchange in the endpoints $x=0$ and $x=1$, we have adiabatic boundary conditions

$$
\frac{\partial \psi}{\partial x}(0, t)=\frac{\partial \psi}{\partial x}(1, t)=0 .
$$

The initial condition $\psi(x, 0)=\phi_{0}(x)$ is chosen from the class $\mathcal{A}$. The solution can be written as follows

$$
\psi(x, t)=\sum_{n=0}^{\infty} c_{n} e^{-n^{2} \pi^{2} \lambda t} \cos (n \pi x),
$$

where the coefficients $c_{n}$, are determined by the initial condition written as a cosine Fourier series

$$
\phi_{0}(x)=\sum_{n=0}^{\infty} c_{n} \cos (n \pi x) .
$$

Suppose the system is perturbed in time instants $t_{1}, t_{2}, \ldots$ through a nonlinear process described below. Being the temperature distribution along the wire initially given by the function $\psi_{0}(x, t)$, for $t_{0}<t<t_{1}$, after the perturbation the temperature function is $\psi_{1}(x, t)$, for $t>t_{1}$. We have continuous time evolution for $t \in] t_{j}, t_{j+1}$ [ and discrete time evolution for $t=t_{j}$. We assume that the perturbation is characterized by an nonlinear map $f$ so that $\psi_{j+1}\left(x, t_{j+1}\right)=f\left(\psi_{j}\left(x, t_{j+1}\right)\right)$, with $\psi_{1}\left(x, t_{1}\right)=f\left(\psi_{0}\left(x, t_{1}\right)\right)$. For simplicity, we choose the time instants to be $t_{k}=k \in \mathbb{N}$. Therefore, the time evolution of the system is described by the sequence of functions

$$
\left\{\psi_{0}, \psi_{1}, \psi_{2}, \ldots, \psi_{k}, \ldots\right\},
$$

each function $\psi_{k}$ satisfying the heat equation for $x \in[0,1], t \in[k, k+1[$ and $k \in \mathbb{N}_{0}$, with initial conditions determined by

$$
\psi_{k+1}(x, k+1)=f\left(\psi_{k}(x, k+1)\right), \text { for } k \in \mathbb{N}_{0},
$$

and $\psi_{0}(x, 0)=\phi_{0}(x)$, a given function from $\mathcal{A}$. 
Example 2. Consider $f_{\mu}(x)=1-\mu x^{2}$, with $\mu=1.7548 \ldots, \lambda=0.00005$ and

$$
\begin{aligned}
\psi_{0}(x, 0)= & 0.2+0.1 \cos (\pi x)-0.2 \cos (2 \pi x)+0.1 \cos (3 \pi x)+ \\
& +0.1 \cos (4 \pi x)-0.1 \cos (5 \pi x)+0.2 \cos (6 \pi x)
\end{aligned}
$$

In the Figure 2, we show the evolution of the system described by the heat equation, which is perturbed in time instants $t=1,2,3,4,56,57,58,59,60$.

The discrete dynamical system used in this work is the following. We consider the state space $\mathcal{A}$, the operator $T_{f_{\mu}}$, and an operator $U_{\lambda, \varepsilon}: \mathcal{A} \rightarrow \mathcal{A}$ which gives the time evolution under the unperturbed regime, with diffusion coefficient $\lambda$. The operator $U_{\lambda, \varepsilon}$ is defined implicitly by

$$
U_{\lambda, \varepsilon} \psi(x, t):=\psi(x, t+\varepsilon) .
$$

Let us consider the operator $V_{\mu, \lambda, \varepsilon}: \mathcal{A} \rightarrow \mathcal{A}$ defined by

$$
V_{\mu, \lambda, \varepsilon}:=T_{f_{\mu}} \circ U_{\lambda, \varepsilon}
$$

Now, since the system is periodically perturbed in natural time instants, it is sufficient to consider a natural value for $\varepsilon$. Therefore, we set $\varepsilon=1$ and we define $V_{\mu, \lambda} \equiv V_{\mu, \lambda, 1}$. Our discrete dynamical system is, then, defined by the pair $\left(\mathcal{A}, V_{\mu, \lambda}\right)$. When we iterate a function $\phi_{0}(x)$ in $\mathcal{A}$, under $V_{\mu, \lambda}$, the obtained iterates $\phi_{k}(x)=V_{\mu, \lambda}^{k}\left(\phi_{0}(x)\right)$ will correspond to the solution given by the sequence of functions (5) in the time instants $\phi_{k}(x)=\psi_{k}(x, k)$. If, for some reason, we need to obtain the temperature function at a non integer time instant $t^{\prime}$ we simply use the solution presented in (3) with initial condition given by $\psi(x, 0)=V_{\mu, \lambda}^{k}\left(\phi_{0}(x)\right)$, where $k=\left[t^{\prime}\right]$ is the integer part of $t^{\prime}$. Then, we evaluate the function for the time instant $t^{\prime}-k$, that is, $\psi\left(x, t^{\prime}-k\right)$.

\section{The Basin of Attraction of $f_{\mu}$}

With the aim of characterizing the evolution of the critical points of the iterates $\phi_{k}=V_{\mu, \lambda}^{k}\left(\phi_{0}\right)$ through a discretization with topological meaning, we define a symbolic coding of the evolution of critical points using the basin of attraction of $f_{\mu}$. So, we define a correspondence between each iterate $\phi_{k}$ and a sequence of finite states (colours) ordered according to the position of each critical point in the basin of attraction.

Let $J_{C}$ be the interval of points $x \in I$ for which $\left|f_{\mu}^{\prime}(x)\right| \leq 1$. Under iteration of $f_{\mu}$ every orbit of an initial point in $J_{C}$ will approach the critical orbit. The 


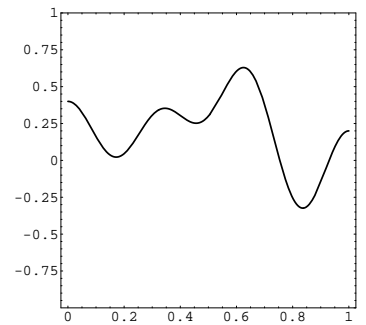

(a)

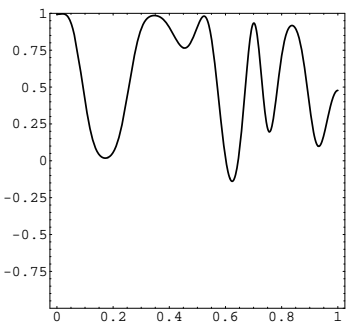

(d)

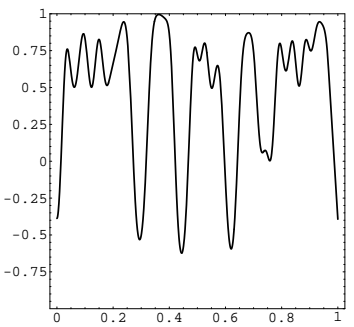

(g)

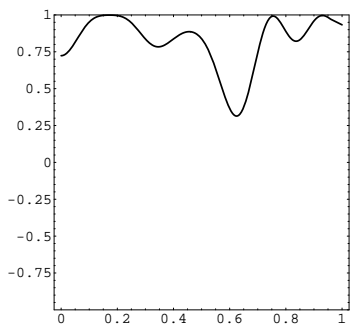

(b)

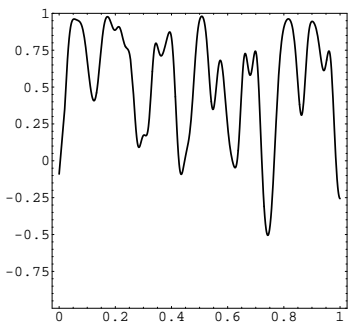

(e)

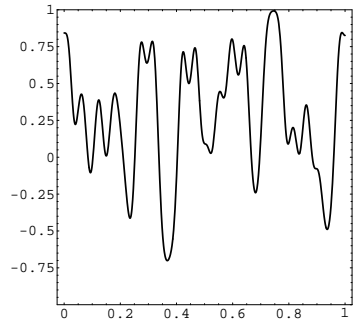

(h)

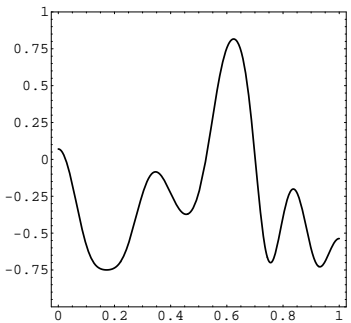

(c)

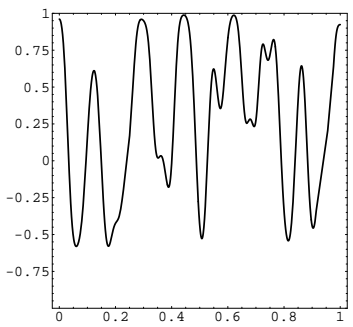

(f)

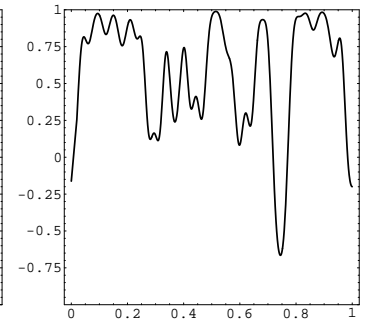

(i)

Figure 2: Graphs of (a) $\psi_{0}(x, 1),(\mathrm{b}) \psi_{1}(x, 2)$, (c) $\psi_{2}(x, 3)$, (d) $\psi_{3}(x, 4),(\mathrm{e}) \psi_{55}(x, 56)$, (f) $\psi_{56}(x, 57),(\mathrm{g}) \psi_{57}(x, 58),(\mathrm{h}) \psi_{58}(x, 59)$ and (i) $\psi_{59}(x, 60)$, with $\lambda=0.00005, f_{\mu}(x)=1-\mu x^{2}, \mu=1.7548 \ldots$ and $\psi_{0}(x, 0)=0.2+0.1 \cos (\pi x)-0.2 \cos (2 \pi x)+0.1 \cos (3 \pi x)+$ $0.1 \cos (4 \pi x)-0.1 \cos (5 \pi x)+0.2 \cos (6 \pi x)$.

same is true for the pre-images of the interval $J_{C}$ which can be codified through the address map. That is, we need the following intervals

$$
J_{S_{1} \ldots S_{k} C}:=I_{S_{1} \ldots S_{k}} \cap f_{\mu}^{-k}\left(J_{C}\right)
$$




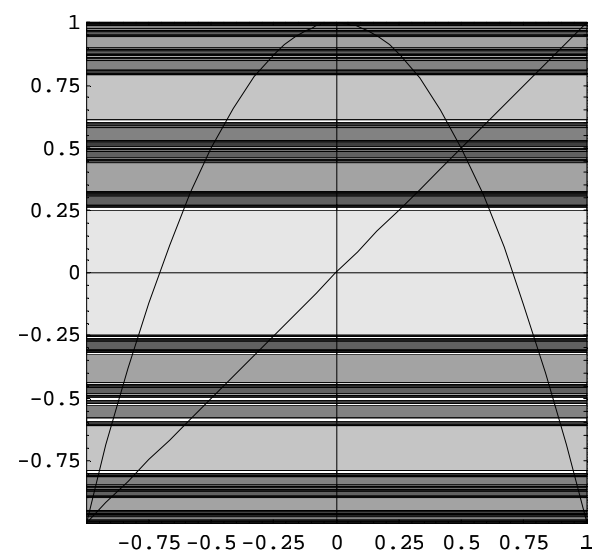

(a)

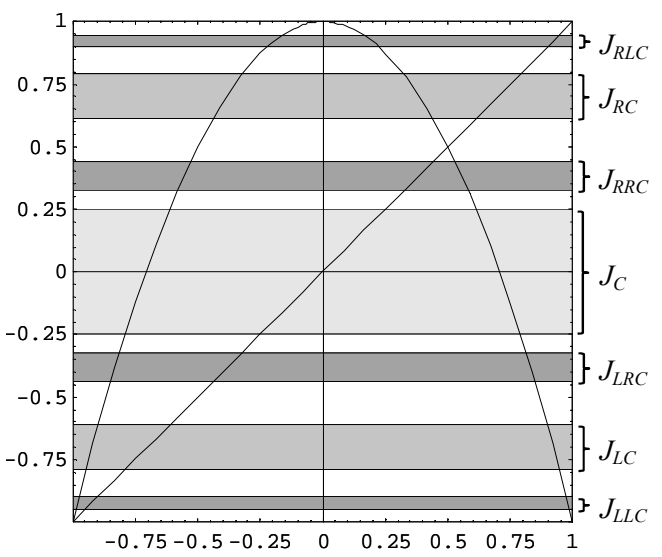

(b)

Figure 3: (a) The basin of attraction of $f_{\mu}(x)=1-\mu x^{2}$, when $\mu=2$, and (b) representation of first intervals of the basin in (a).

These intervals gives an underlying structure, or grid, which will be our reference to define the codification of the orbits under the operator $V_{\mu, \lambda}$. These intervals depend on the parameter $\mu$ and the kneading sequence of $f_{\mu}$, since if a sequence $S_{1} \ldots S_{k}$ is non admissible then $J_{S_{1} \ldots S_{k} C}=\varnothing$. Moreover, we have to be careful to use the intervals $J_{S_{1} \ldots S_{k} C}$ since they eventually overlap.

In next figures, we present the basin of attraction of $f_{\mu}$, in the first one for $\mu=2$ and in the second for $\mu=1.7548 \ldots$ and $\mu=1.3815 \ldots$, respectively (the parameter $\mu=1.7548 \ldots$ corresponds to a period three and the parameter $\mu=$ $1.3815 \ldots$ corresponds to a period eight). Note that to each colour corresponds a different level of pre-images (from lighter to darker) regarding to the interval of attraction, $J_{C}$. The basin of attraction is given by

$$
\Lambda=J_{C} \cup J_{L C} \cup J_{R C} \cup J_{L L C} \cup J_{L R C} \cup J_{R R C} \cup J_{R L C} \cup \cdots .
$$

Now, we present the construction of the basin of attraction of $f_{\mu}$, when $\mu=2$. For other values of the parameter $\mu$, the construction is similar.

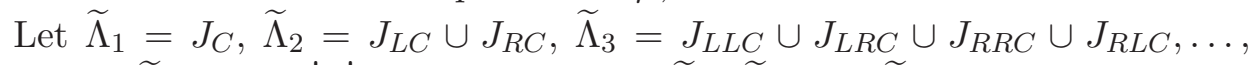
in general $\widetilde{\Lambda}_{k}=\bigcup_{S \in\{L, R\}^{k-1}} J_{S C}$ (the sets $\widetilde{\Lambda}_{1}, \widetilde{\Lambda}_{2}$ and $\widetilde{\Lambda}_{3}$ are represented in Figure $3(\mathrm{~b}))$, and let be $U_{1}=U_{2}=U_{3}=\varnothing, \ldots, U_{k}=\bigcup J_{S C}$, where $S \in\{L, R\}^{k-1}$ 


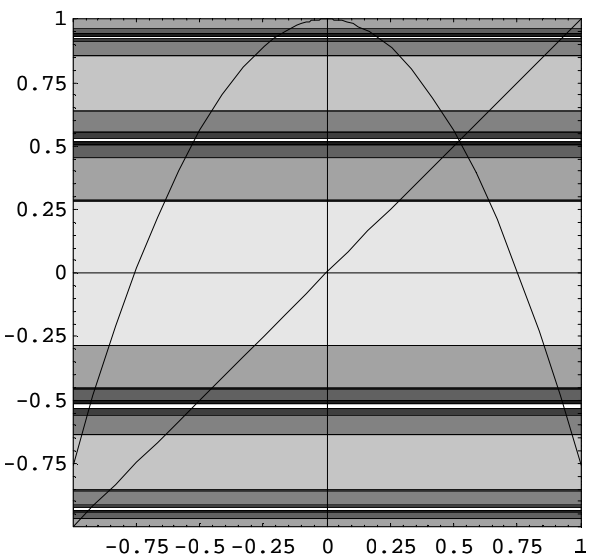

(a)

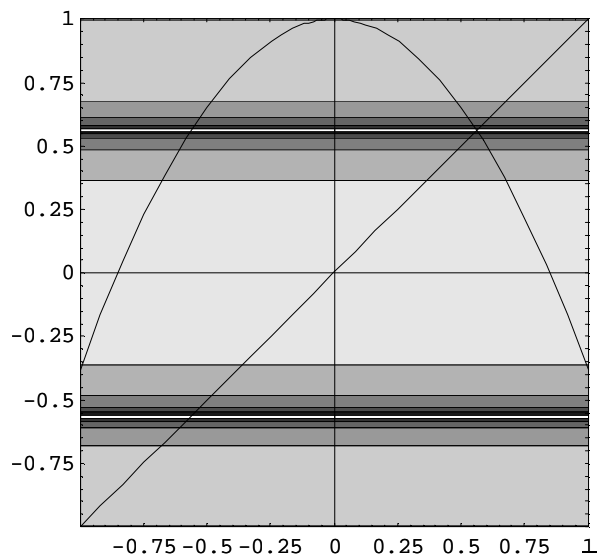

(b)

Figure 4: The basin of attraction of $f_{\mu}(x)=1-\mu x^{2}$, when (a) $\mu=$ $1.7548 \ldots$ and (b) $\mu=1.3815 \ldots$.

$S \in\{L, R\}^{k-1}$ and such that $f_{\mu}\left(J_{S C}\right) \cap \bigcup_{j=1}^{k-1} \widetilde{\Lambda}_{j} \neq \varnothing$. Then, considering $\Lambda_{k}=$ $\widetilde{\Lambda}_{k}-U_{k}$, the basin of attraction of $f_{\mu}$ is given by $\Lambda=\bigcup_{k=1} \Lambda_{k}$.

We can, after a systematic analysis, determine the following

$$
\begin{gathered}
U_{1}=\varnothing, U_{2}=\varnothing, U_{3}=\varnothing, \\
U_{4}=J_{L R L C} \cup J_{R R L C}, \\
U_{5}=J_{L L R L C} \cup J_{L R R L C} \cup J_{L R L L C} \cup J_{R R L L C} \cup J_{R R R L C} \cup J_{R L R L C}, \\
U_{6}=J_{L L L R L C} \cup J_{L L R R L C} \cup J_{L L R L L C} \cup J_{L R R L L C} \cup J_{L R R R L C} \cup \\
\cup J_{L R L R L C} \cup J_{L R L R R C} \cup J_{L R L L R C} \cup J_{L R L L L C} \cup J_{R R L L L C} \cup \\
\cup J_{R R L L R C} \cup J_{R R L R R C} \cup J_{R R L R L C} \cup J_{R R R R L C} \cup J_{R R R L L C} \cup \\
\cup J_{R L R L L C} \cup J_{R L R R L C} \cup J_{R L L R L C}
\end{gathered}
$$

and

$$
\begin{aligned}
U_{7}= & J_{L L L L R L C} \cup J_{L L L R R L C} \cup J_{L L L R L L C} \cup J_{L L R R L L C} \cup \\
& \cup J_{L L R R R L C} \cup J_{L L R L R L C} \cup J_{L L R L R R C} \cup J_{L L R L L R C} \cup
\end{aligned}
$$




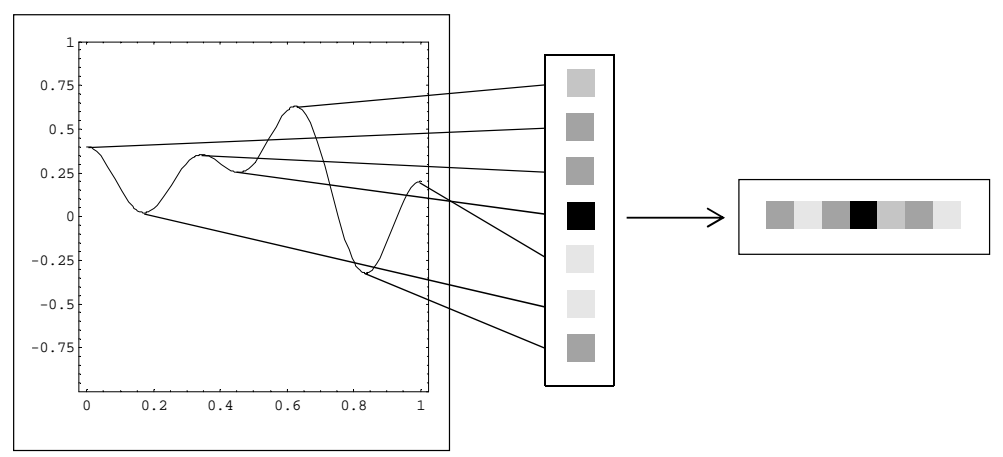

Figure 5: Correspondence between the critical points of $\psi_{0}(x, 0)=0.2+$ $0.1 \cos (\pi x)-0.2 \cos (2 \pi x)+0.1 \cos (3 \pi x)+0.1 \cos (4 \pi x)-0.1 \cos (5 \pi x)+$ $0.2 \cos (6 \pi x)$ and the sequence of colours obtained by the basin of attraction of the quadratic map $f_{\mu}$, with $\mu=2$.

$$
\begin{aligned}
& \cup J_{L L R L L L C} \cup J_{L R R L L L C} \cup J_{L R R L L R C} \cup J_{L R R L R R C} \cup \\
& \cup J_{L R R L R L C} \cup J_{L R R R R L C} \cup J_{L R R R L R C} \cup J_{L R R R L L C} \cup \\
& \cup J_{L R L R L L C} \cup J_{L R L R L R C} \cup J_{L R L R R L C} \cup J_{L R L L R L C} \cup \\
& \cup J_{L R L L R R C} \cup J_{L R L L L R C} \cup J_{L R L L L L C} \cup J_{R R L L L L C} \cup \\
& \cup J_{R R L L L R C} \cup J_{R R L L R R C} \cup J_{R R L L R L C} \cup J_{R R L R R L C} \cup \\
& \cup J_{R R L R L R C} \cup J_{R R L R L L C} \cup J_{R R R R L L C} \cup J_{R R R R L R C} \cup \\
& \cup J_{R R R R R L C} \cup J_{R R R L R L C} \cup J_{R R R L R R C} \cup J_{R R R L L R C} \cup \\
& \cup J_{R R R L L L C} \cup J_{R L R L L L C} \cup J_{R L R L L R C} \cup J_{R L R L R R C} \cup \\
& \cup J_{R L R L R L C} \cup J_{R L R R R L C} \cup J_{R L R R L L C} \cup J_{R L L R L L C} \cup \\
& \cup J_{R L L R R L C} \cup J_{R L L L R L C} \cdot
\end{aligned}
$$

Now, we define a symbolic coding, that is, to each $\Lambda_{k}$, with $k=1, \ldots, 7$, we associate the symbol $k$, and to $\Lambda-\bigcup_{k=1}^{7} \Lambda_{k}$ we associate the symbol 8. Finally, to each symbol we associate a different colour, from white for symbol 1 to black for symbol 8.

The Figure 5 shows an example of the correspondence between the critical points and the sequence of colours. 


\section{The Evolution of Critical Points of the Iterates $\phi_{k}=V_{\mu, \lambda}^{k}\left(\phi_{0}\right)$ for Positive Topological Entropy of $f_{\mu}$}

In the work of Correia et al. [1] was established that, in certain conditions - namely positive topological entropy of $f_{\mu}$ - the iterates, under $V_{\mu, \lambda}$, have an exponential grow of number of critical points up to a certain level. After attaining a certain number of critical points, which depends on the parameters, this number oscillates and becomes limited.

As we referred above, the particular characteristic of the system $\left(\mathcal{A}, T_{f}\right)$, for certain choices of the map $f$, is that the number of different critical values will always grow with the iteration of $T_{f}$, for almost all initial conditions. On the contrary, for the dynamical system $\left(\mathcal{A}, V_{\mu, \lambda}\right)$, the number of critical points does not grow exponentially. Indeed, it attains a balance between the creation of new critical points, due to the interval map effect, and the destruction of critical points, due to the dissipative effect of the heat equation.

In what following, we present some examples with the evolution of critical points using the coding defined above. For these examples, we consider the following initial conditions

$$
\begin{aligned}
& \psi_{0}(x)= 0.2+0.1 \cos (\pi x)-0.2 \cos (2 \pi x)+0.1 \cos (3 \pi x)+ \\
&+0.1 \cos (4 \pi x)-0.1 \cos (5 \pi x)+0.2 \cos (6 \pi x), \\
& \widetilde{\psi}_{0}(x)=0.2+0.1 \cos (\pi x)-0.2 \cos (2 \pi x)+0.1 \cos (3 \pi x), \\
& \varphi_{0}(x)=0.2+0.1 \cos (\pi x)-0.2 \cos (2 \pi x)+0.1 \cos (3 \pi x)+ \\
&+0.1 \cos (4 \pi x)-0.1 \cos (5 \pi x)+0.2 \cos (6 \pi x)+ \\
&+0.1 \cos (7 \pi x)+0.2 \cos (8 \pi x)+0.2 \cos (9 \pi x), \\
& \widetilde{\varphi}_{0}(x)=0.3 \cos (2 \pi x)+0.2 \cos (4 \pi x)-0.2 \cos (6 \pi x)+ \\
& \quad-0.3 \cos (29 \pi x), \\
& \phi_{0}(x)=\quad 0.07 \cos (2 \pi x)+0.07 \cos (3 \pi x)-0.07 \cos (5 \pi x)+ \\
&+0.07 \cos (6 \pi x)-0.07 \cos (29 \pi x), \\
& \widetilde{\phi}_{0}(x)=0.03 \cos (2 \pi x)+0.05 \cos (3 \pi x)-0.07 \cos (4 \pi x)+ \\
&+0.07 \cos (5 \pi x)+0.03 \cos (7 \pi x),
\end{aligned}
$$




$$
\rho_{0}(x)=0.45-0.45 \cos (2 \pi x)
$$

and

$$
\widetilde{\rho}_{0}(x)=-0.75 \cos (4 \pi x) .
$$

Considering $f_{\mu}(x)=1-\mu x^{2}$, with $\mu=2, \lambda=0.00005$ and $\psi_{0}(x, 0)=\psi_{0}(x)$ the symbolic evolution of critical points, for first five iterates, is given by

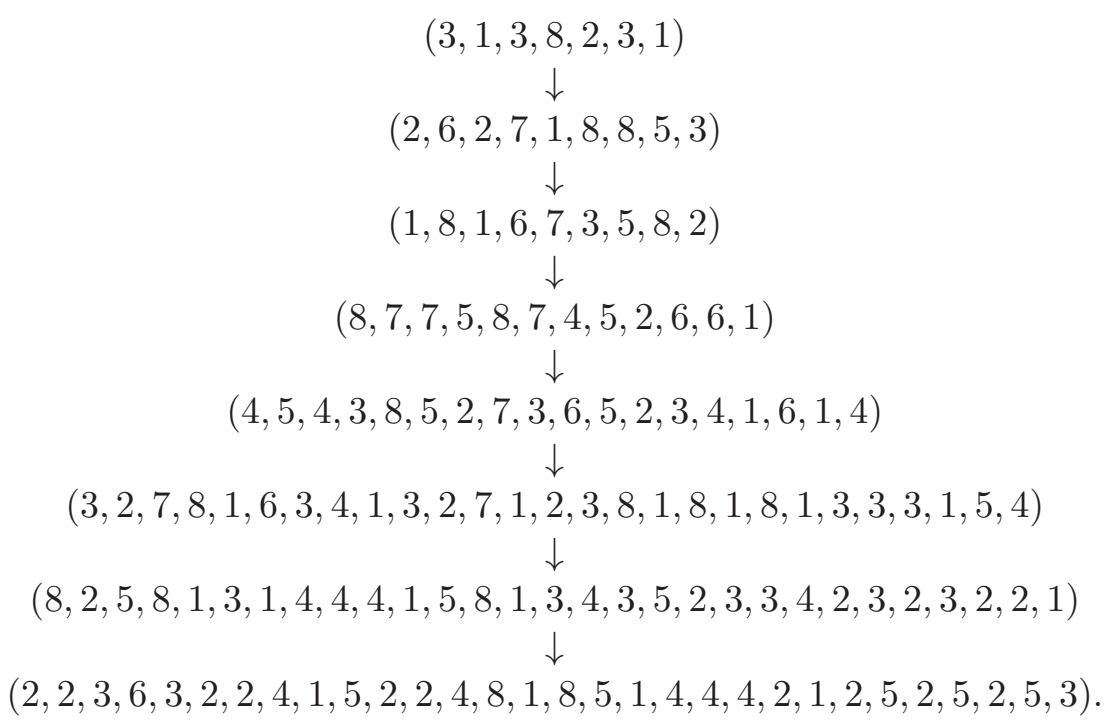

In Figure 6, and for previous functions and parameters, we show the corresponding evolution of critical points of $V_{\mu, \lambda}^{k}\left(\psi_{0}(x)\right)$, with $k=0, \ldots, 99$. We present the correspondence between some iterates and the lines of colours.

In Figures 7 and 8, we present similar graphs, for $\mu=1.7548$. Note that in the last figure the symmetry of functions is maintained over time.

As we can see in the Table 1, the average number of critical points of the iterates depends on the diffusion coefficient $\lambda$ and the parameter $\mu$. When we decrease the diffusion coefficient $\lambda$, the average number of critical points, at which the temperature function stabilizes, increases. Also, when we increase the parameter $\mu$, the average number of critical points increases.

Using the values obtained numerically for the distribution of the critial points, we are led to the following result.

Numerical Result 1. When $\mu$ is such that $h_{t}\left(f_{\mu}\right)>0$, the average of critical points, $\eta(\lambda)$, does not depend significatly on $\mu$, only on $\lambda$. Moreover, if $\lambda \in[0.0000075,0.01]$, then we have aproximately the rule

$$
\eta(\lambda)=C_{0} \lambda^{C_{1}}
$$




\begin{tabular}{|c|c|c|c|c|c|c|c|c|c|c|}
\hline \multirow{4}{*}{ 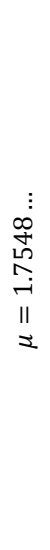 } & \multirow{2}{*}{$\begin{array}{l}\text { L } \\
8 \\
0 \\
0 \\
0 \\
\text { II } \\
\sim\end{array}$} & 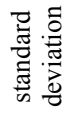 & $\stackrel{\text { of }}{\tilde{m}}$ & r) & $\begin{array}{l}\text { ते } \\
\text { i }\end{array}$ & $\overrightarrow{\dot{m}}$ & $\underset{m}{\vec{m}}$ & 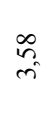 & $\stackrel{\substack{n \\
m}}{\stackrel{n}{n}}$ & $\begin{array}{l}\infty \\
\text { of }\end{array}$ \\
\hline & & 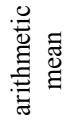 & $\begin{array}{l}\hat{o} \\
\text { o } \\
i\end{array}$ & $\begin{array}{l}\overrightarrow{0} \\
\infty \\
\text { i }\end{array}$ & $\begin{array}{l}\text { oे } \\
\text { o } \\
\text { in }\end{array}$ & $\begin{array}{l}n \\
\tilde{n} \\
\stackrel{\sim}{v}\end{array}$ & $\begin{array}{l}\vec{\infty} \\
\stackrel{\sim}{N}\end{array}$ & $\begin{array}{l}\hat{\Omega} \\
\hat{\sim}\end{array}$ & $\begin{array}{l}\text { fo } \\
\text { if }\end{array}$ & $\begin{array}{l}\text { ते } \\
\infty \\
\text { in }\end{array}$ \\
\hline & \multirow{2}{*}{$\begin{array}{l}\overrightarrow{8} \\
8 \\
0 \\
0 \\
0 \\
\text { II } \\
\sim\end{array}$} & 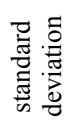 & $\begin{array}{l}\underset{+}{+} \\
+\end{array}$ & $\begin{array}{l}\stackrel{0}{f} \\
\stackrel{f}{*}\end{array}$ & $\begin{array}{l}\vec{\infty} \\
+\end{array}$ & $\begin{array}{l} \pm \\
\forall\end{array}$ & $\stackrel{\text { I }}{\underset{f}{*}}$ & ్ㅗㄱ & క్ర & $\begin{array}{l}n \\
\tilde{m}\end{array}$ \\
\hline & & 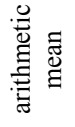 & $\underset{\tilde{b}}{\tilde{b}}$ & $\begin{array}{l}n \\
\infty \\
8 \\
8\end{array}$ & ले & $\begin{array}{l}\infty \\
\stackrel{0}{8}\end{array}$ & $\begin{array}{l}\text { J } \\
\text { si }\end{array}$ & $\begin{array}{l}\hat{\text { }} \\
\hat{\sigma}\end{array}$ & $\begin{array}{l}\infty \\
\text { î } \\
\text { di }\end{array}$ & $\begin{array}{l}\text { n. } \\
\text { î }\end{array}$ \\
\hline \multirow{4}{*}{$\begin{array}{l}\text { N } \\
\text { II } \\
\text { Z }\end{array}$} & \multirow{2}{*}{$\begin{array}{l}\text { L } \\
0 \\
0 \\
0 \\
0 \\
\text { II } \\
\sim\end{array}$} & 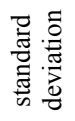 & $\begin{array}{l}\infty \\
\infty \\
i\end{array}$ & $\begin{array}{l}n \\
\infty \\
i\end{array}$ & mे & $\vec{m}$ & $\hat{n}$ & in & $\begin{array}{l}\text { s. } \\
\text { in }\end{array}$ & $\begin{array}{c}\infty \\
\infty \\
\infty \\
\infty\end{array}$ \\
\hline & & 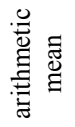 & $\frac{n}{\hat{i}}$ & $\begin{array}{l}\text { J } \\
\text { ते }\end{array}$ & $\stackrel{\check{i}}{\stackrel{2}{2}}$ & $\begin{array}{l}\text { q } \\
\text { নे }\end{array}$ & $\begin{array}{l}\text { ஸे } \\
\text { ते }\end{array}$ & $\begin{array}{l}\tilde{2} \\
\text { ते }\end{array}$ & $\begin{array}{l}\infty \\
\infty \\
\stackrel{\lambda}{~}\end{array}$ & $\begin{array}{l}\stackrel{0}{\circ} \\
\stackrel{n}{m}\end{array}$ \\
\hline & \multirow{2}{*}{$\begin{array}{l}\overrightarrow{0} \\
0 \\
0 \\
0 \\
\text { II } \\
\sim\end{array}$} & 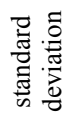 & $\begin{array}{c}m \\
i\end{array}$ & $\begin{array}{l}\infty \\
\infty \\
+\end{array}$ & $\begin{array}{l}\tilde{n} \\
n \\
n\end{array}$ & $\begin{array}{l}\text { जे } \\
\text { in }\end{array}$ & \begin{tabular}{l}
$\mathcal{W}$ \\
\multirow{+}{*}{}
\end{tabular} & $\begin{array}{l}n \\
b \\
+\end{array}$ & $\begin{array}{l}\infty \\
\infty \\
0\end{array}$ & $\begin{array}{l} \pm \\
\stackrel{0}{n}\end{array}$ \\
\hline & & 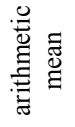 & $\begin{array}{l}\infty \\
\text { से }\end{array}$ & $\frac{\hat{\sigma}}{3}$ & 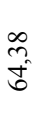 & $\begin{array}{l}0 \\
i \\
\hat{b}\end{array}$ & $\begin{array}{l}\text { o } \\
\text { tే }\end{array}$ & $\begin{array}{l}\vec{F} \\
\text { f }\end{array}$ & $\hat{n}$ & $\begin{array}{l}\infty \\
0 \\
0 \\
0\end{array}$ \\
\hline \multicolumn{3}{|c|}{ 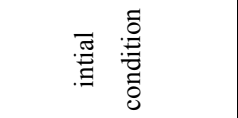 } & $\$^{0}$ & $12^{\circ}$ & $\stackrel{0}{0}$ & $1 Q^{\circ}$ & 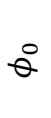 & 10 & 2 & $10^{\circ}$ \\
\hline
\end{tabular}

Table 1: The arithmetic mean and the standard deviation for eight different initial conditions, $\psi_{0}, \widetilde{\psi}_{0}, \varphi_{0}, \widetilde{\varphi}_{0}, \phi_{0}, \widetilde{\phi}_{0}, \rho_{0}, \widetilde{\rho}_{0}$, of the number of critical points. The values are presented for two different values of parameter $\mu(\mu=2$ and $\mu=1.7548 \ldots)$ and for two different values of the diffusion coefficient $\lambda(\lambda=0.00005$ and $\lambda=0.00001)$. 
with $C_{0}=0.25 \pm 0.05$ and $C_{1}=-0.485 \pm 0.015$ (see Figure 9).

\section{The Evolution of Critical Points of the Iterates $\phi_{k}=V_{\mu, \lambda}^{k}\left(\phi_{0}\right)$ for Topological Entropy of $f_{\mu}$ Equal to Zero}

For the cases in which the quadratic map $f_{\mu}$ has topological entropy equal to zero, that is, that for the cases in $\mu$ is such that $h_{t}\left(f_{\mu}\right)=0$, it is not possible to present a formula similar to (6), since the number and evolution of critical points depends strongly on the initial conditions, and the standard deviation is very high although the growth is low, see Table 2 .

In Figures 10 and 11, we present the evolution of critical points of $V_{\mu, \lambda}^{k}\left(\psi_{0}(x)\right)$, with $k=0, \ldots, 99$, and the correspondence between some iterates and the lines of colours, for $\mu=1.3815 \ldots$, which corresponds to a period eight. 


\begin{tabular}{|c|c|c|c|c|}
\hline \multirow{3}{*}{$\begin{array}{c}\text { intial } \\
\text { condition }\end{array}$} & \multicolumn{4}{|c|}{$\mu=1.3815 \ldots$} \\
\hline & \multicolumn{2}{|c|}{$\lambda=0.00001$} & \multicolumn{2}{|c|}{$\lambda=0.00005$} \\
\hline & $\begin{array}{l}\text { arithmetic } \\
\text { mean }\end{array}$ & $\begin{array}{l}\text { standard } \\
\text { deviation }\end{array}$ & $\begin{array}{c}\text { arithmetic } \\
\text { mean }\end{array}$ & $\begin{array}{l}\text { standard } \\
\text { deviation }\end{array}$ \\
\hline$\psi_{0}$ & 28,23 & 5,50 & 16,55 & 2,28 \\
\hline$\widetilde{\psi}_{0}$ & 29,76 & 9,71 & 13,55 & 3,25 \\
\hline$\varphi_{0}$ & 35,14 & 3,45 & 15,11 & 3,09 \\
\hline$\tilde{\varphi}_{0}$ & 41,09 & 6,52 & 15,37 & 5,95 \\
\hline$\phi_{0}$ & 22,61 & 7,51 & 14,41 & 6,17 \\
\hline$\tilde{\phi}_{0}$ & 35,58 & 4,06 & 7,75 & 2,46 \\
\hline$\rho_{0}$ & 33,17 & 6,13 & 15,02 & 2,19 \\
\hline$\tilde{\rho}_{0}$ & 43,08 & 9,95 & 23,95 & 6,09 \\
\hline
\end{tabular}

Table 2: The arithmetic mean and the standard deviation for eight different initial conditions, $\psi_{0}, \widetilde{\psi}_{0}, \varphi_{0}, \widetilde{\varphi}_{0}, \phi_{0}, \widetilde{\phi}_{0}, \rho_{0}, \widetilde{\rho}_{0}$, of the number of critical points. The values are presented for parameter $\mu=1.3815 \ldots$, which corresponds to period eight for $f_{\mu}$, and for two different values of the diffusion coefficient $\lambda(\lambda=0.00005$ and $\lambda=0.00001)$. 


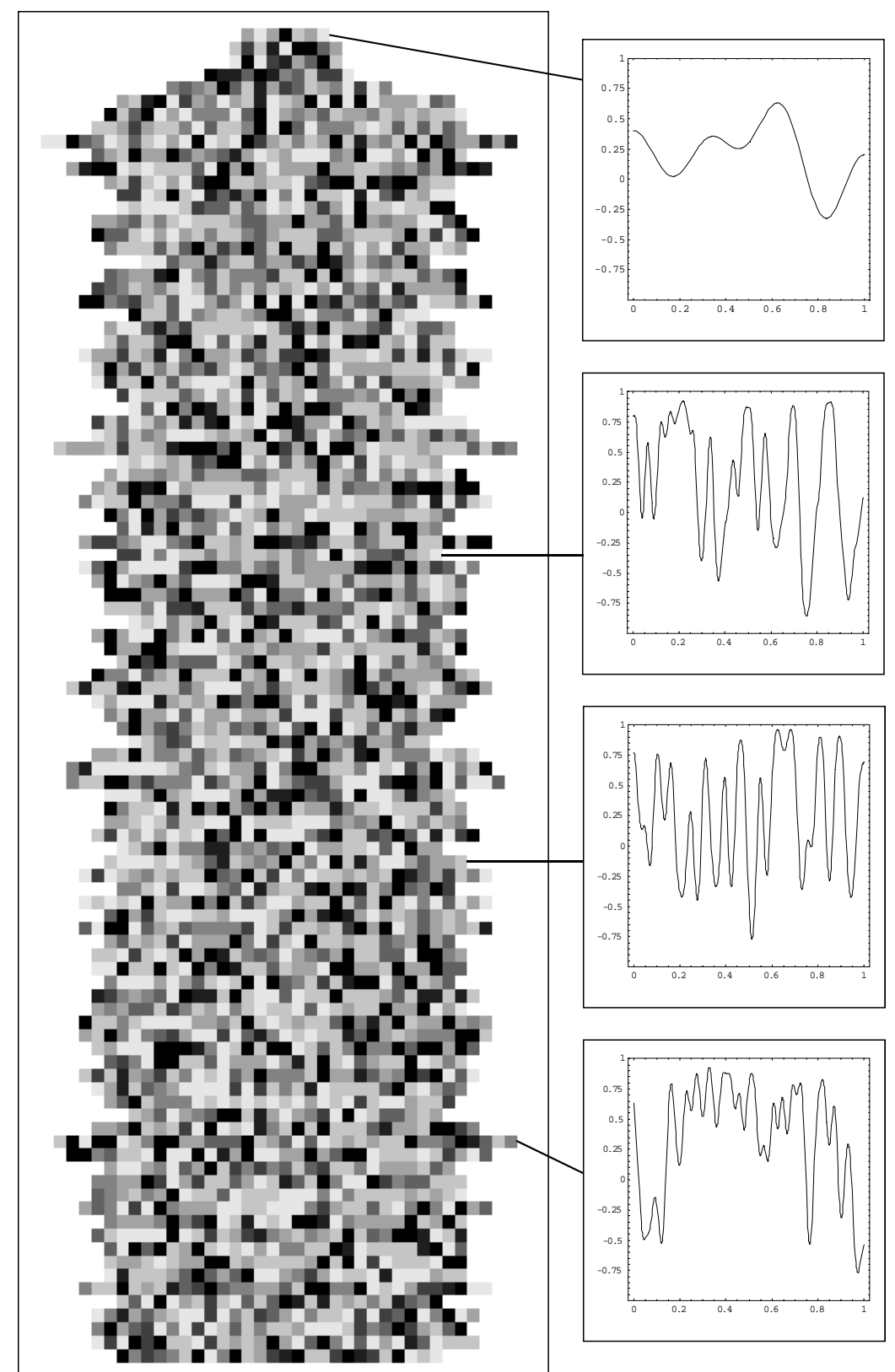

Figure 6: Graph of the evolution of critical points of $V_{\mu, \lambda}^{k}\left(\psi_{0}(x)\right)$, with $k=0, \ldots, 99, \lambda=0.00005, f_{\mu}(x)=1-\mu x^{2}, \mu=2$ and $\psi_{0}(x, 0)=0.2+$ $0.1 \cos (\pi x)-0.2 \cos (2 \pi x)+0.1 \cos (3 \pi x)+0.1 \cos (4 \pi x)-0.1 \cos (5 \pi x)+$ $0.2 \cos (6 \pi x)$. 


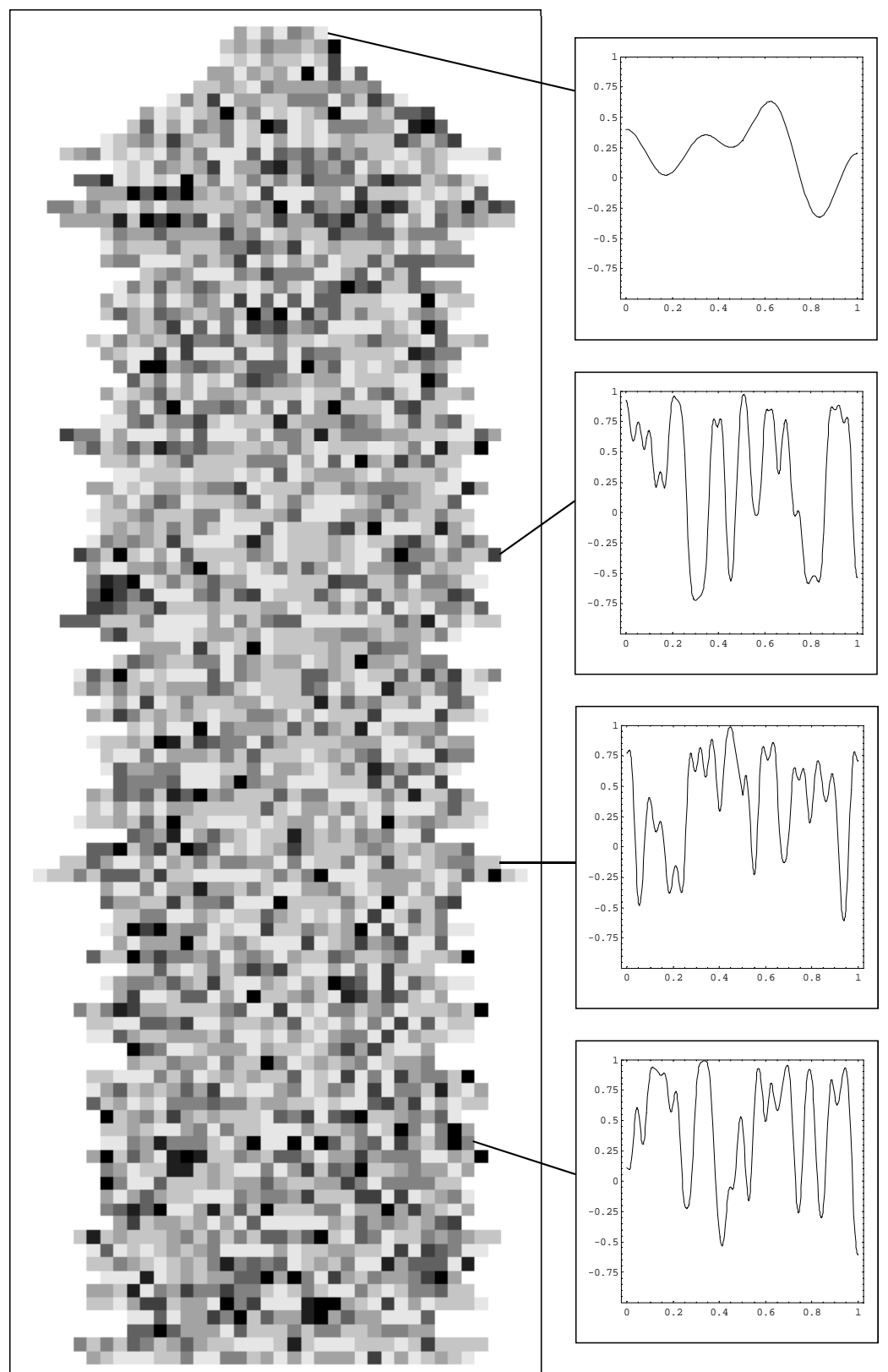

Figure 7: Graph of the evolution of critical points of $V_{\mu, \lambda}^{k}\left(\psi_{0}(x)\right)$, with $k=0, \ldots, 99, \lambda=0.00005, f_{\mu}(x)=1-\mu x^{2}, \mu=1.7548 \ldots$ and $\psi_{0}(x, 0)=0.2+0.1 \cos (\pi x)-0.2 \cos (2 \pi x)+0.1 \cos (3 \pi x)+0.1 \cos (4 \pi x)-$ $0.1 \cos (5 \pi x)+0.2 \cos (6 \pi x)$. 


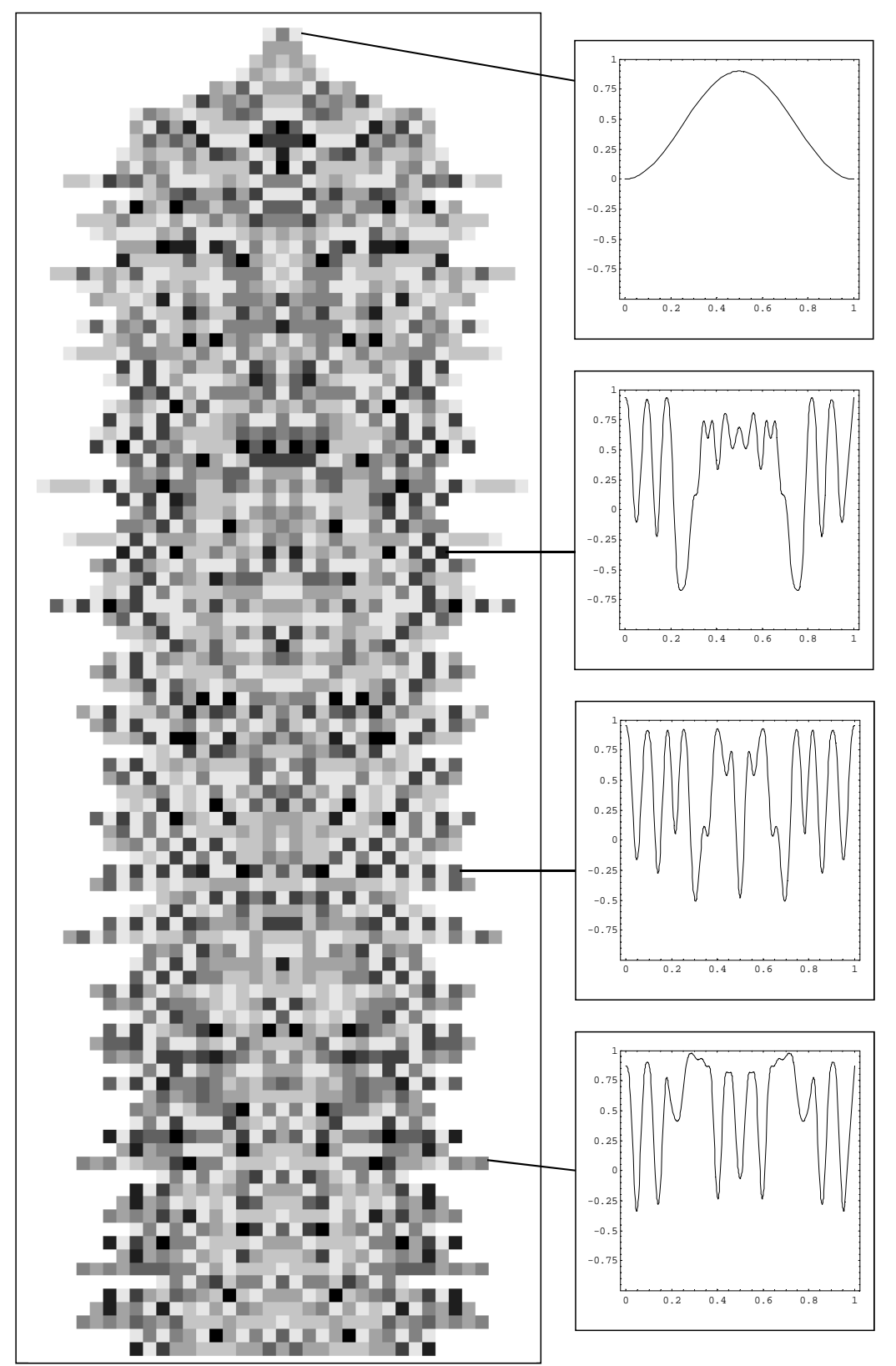

Figure 8: Graph of the evolution of critical points of $V_{\mu, \lambda}^{k}\left(\psi_{0}(x)\right)$, with $k=0, \ldots, 99, \lambda=0.00005, f_{\mu}(x)=1-\mu x^{2}, \mu=1.7548 \ldots$ and $\rho_{0}(x, 0)=0.45-0.45 \cos (2 \pi x)$. 


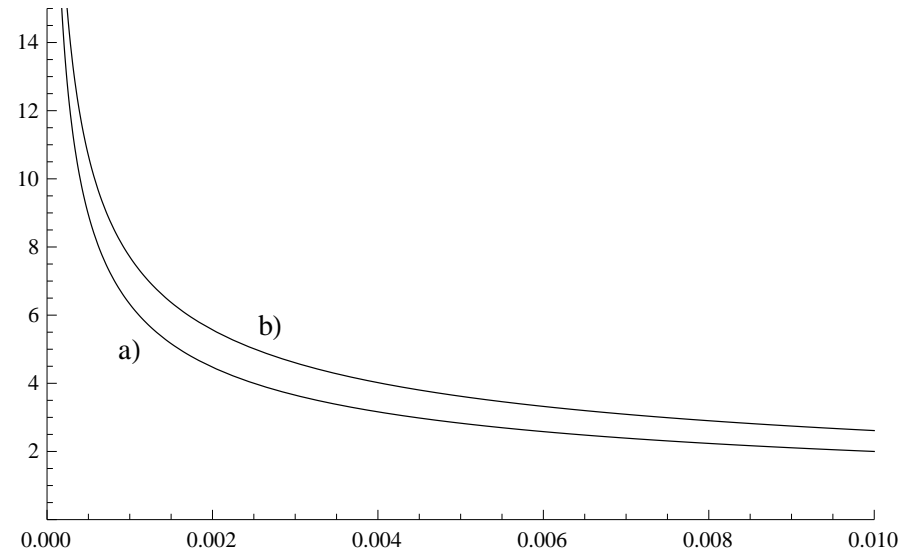

Figure 9: Graph of $\eta(\lambda)=C_{0} \lambda^{C_{1}}$, with a) $C_{0}=0.2$ and $C_{1}=-0.5$ and b) $C_{0}=0.3$ and $C_{1}=-0.47$. 


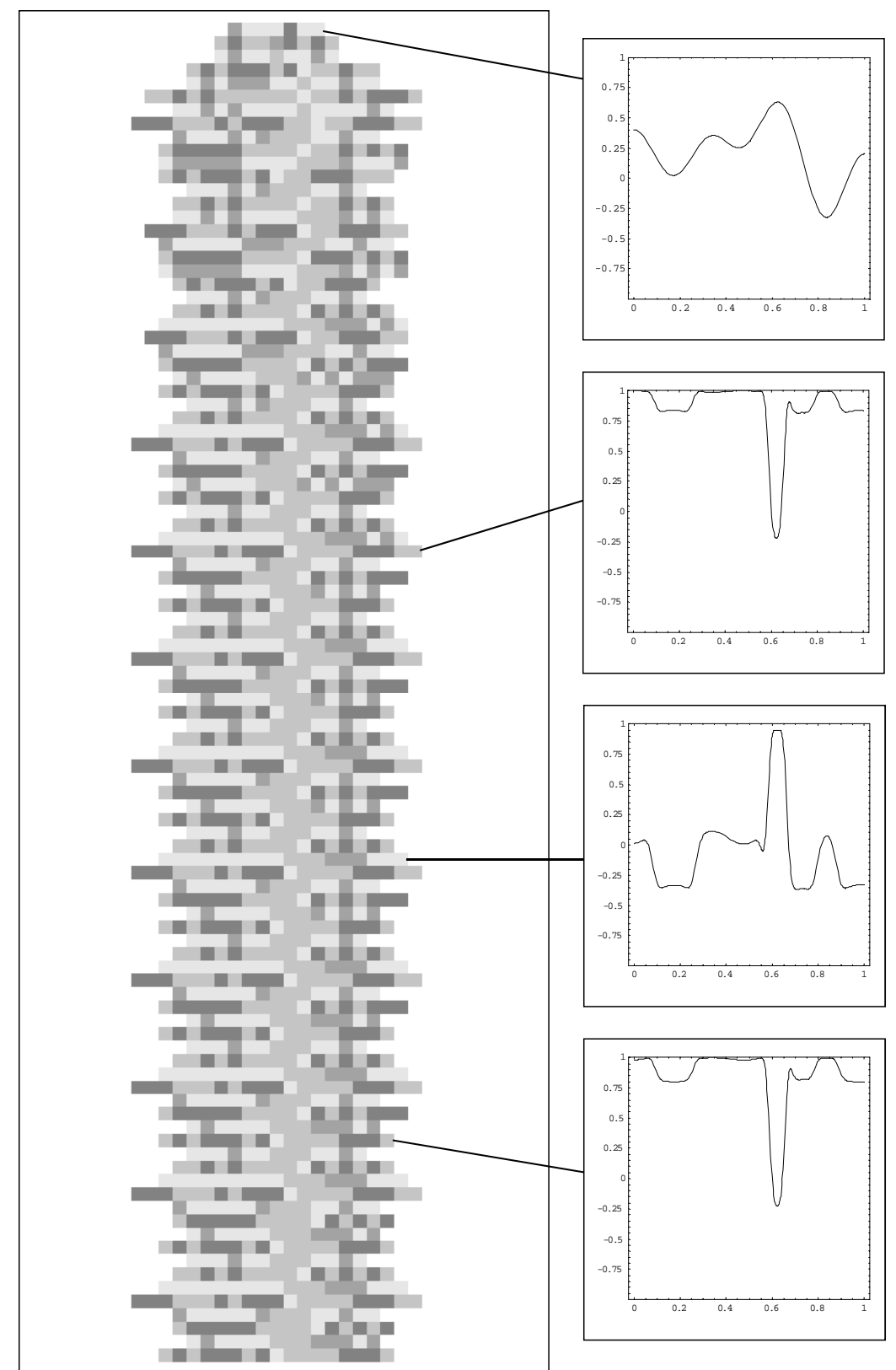

Figure 10: Graph of the evolution of critical points of $V_{\mu, \lambda}^{k}\left(\psi_{0}(x)\right)$, with $k=0, \ldots, 99, \lambda=0.00005, f_{\mu}(x)=1-\mu x^{2}, \mu=1.3815 \ldots$ and $\psi_{0}(x, 0)=0.2+0.1 \cos (\pi x)-0.2 \cos (2 \pi x)+0.1 \cos (3 \pi x)+0.1 \cos (4 \pi x)-$ $0.1 \cos (5 \pi x)+0.2 \cos (6 \pi x)$. 


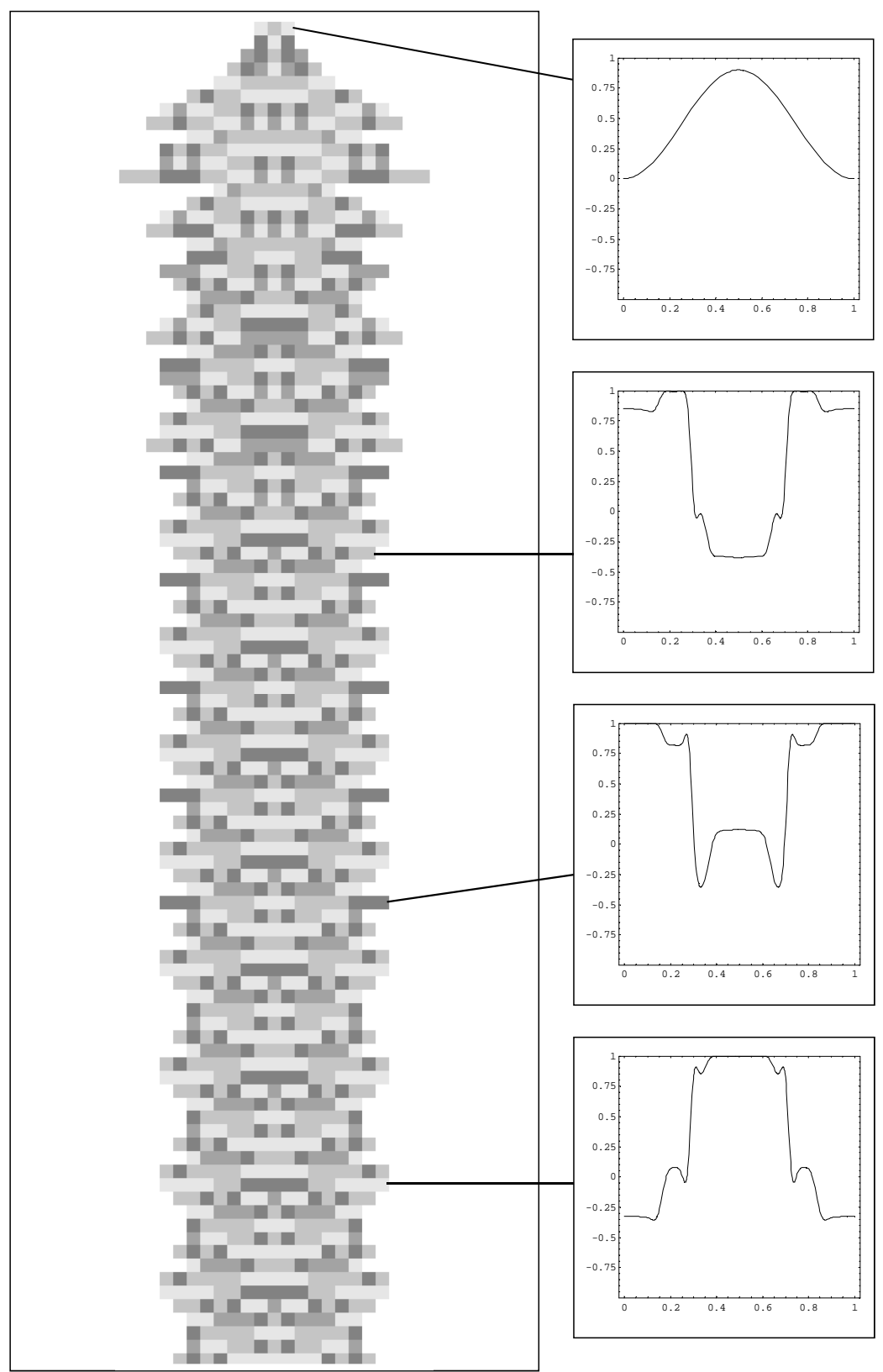

Figure 11: Graph of the evolution of critical points of $V_{\mu, \lambda}^{k}\left(\psi_{0}(x)\right)$, with $k=0, \ldots, 99, \lambda=0.00005, f_{\mu}(x)=1-\mu x^{2}, \mu=1.3815 \ldots$ and $\rho_{0}(x, 0)=0.45-0.45 \cos (2 \pi x)$. 


\section{Acknowledgements}

This work was partially supported by national funds from the FCT - Fundação para a Ciência e a Tecnologia, within the project UID/MAT/04674/2013.

\section{References}

[1] M.F. Correia, C.C. Ramos and S. Vinagre, Nonlinearly perturbed heat equation, Int. J. of Pure and Applied Math., 94, No. 2 (2014), 279-296, doi: 10.12732/ijpam.v92i2.10.

[2] R. Severino, A.N. Sharkovsky, J. Sousa Ramos and S. Vinagre, Symbolic Dynamics in Boundary Value problems, Grazer Math. Ber, 346 (2004), 393-402.

[3] R. Severino, A.N. Sharkovsky, J. Sousa Ramos and S. Vinagre, Topological invariants in a model of a time-delayed Chua's circuit, Nonlinear Dynamics, 44, (2006), 81-90, doi: : 10.1007/s11071-006-1942-4.

[4] R. Severino, A.N. Sharkovsky, J. Sousa Ramos and S. Vinagre, Computing topological invariants in boundary value problems reducible to difference equations, Proceedings of the International Conference on Difference Equations, Special Functions and Orthogonal Polynomials, World Scientific Publishing (2007), 741-751, doi: : 10.1142/9789812770752_0060.

[5] A.N. Sharkovsky, R. Severino and S. Vinagre, Difference Equations and Nonlinear Boundary Value Problems for Hyperbolic Systems, Discrete Dynamics and Difference Equations - Proceedings of the Twelfth International Conference on Difference Equations and Applications, World Scientific Publishing (2010), 400-409, doi: : 10.1142/9789814287654_0034.

[6] S. Vinagre, R. Severino and J. Sousa Ramos, Topological invariants in nonlinear boundary value problems, Chaos Solitons Fractals, 25, (2005), 65-78, doi: : 10.1016/j.chaos.2004.11.043.

[7] A.N. Sharkovsky, Ideal Turbulence, Nonlinear Dynamics, 44 (2006), 15-27, doi: 10.1007/s11071-006-1931-7.

[8] A.N. Sharkovsky, Ideal turbulence and problems of its visualization, Proceedings of the International Conference on Difference Equations, Special Functions and Orthogonal Polynomials, World Scientific Publishing (2007), 617-635, doi: 10.1142/S0218127494000216.

[9] J. Milnor and W. Thurston, On Iterated Maps of the Interval, Proceedings Univ. Maryland (ed. J. C. Alexander), 1986-1987, Lect. Notes in Math., Springer-Verlag, Berlin, New York, 1342 (1988), 465-563, doi: 10.1007/BFb0082847). 
